\title{
Normalizing Abstraction: Interdisciplinarity and Familiarity in Beginning Design Pedagogy
}

\section{ERLENE CLARK}

Austin Community College
In examining the mechanisms by which architecture's curricular core expresses itself, this analysis argues for a rethinking of pedagogical approaches to the understanding of abstraction in beginning design. Humans are continually learning about the world around them through their perception and comparison of new ideas and objects to familiar ones. And yet, beginning design students are often viewed as empty vessels ready to absorb any information regarding conceptual abstraction transmitted to them by design studio critics.

Regarding the development of abstract thinking skills, students can build on familiar, yet potentially underutilized, cognitive skills they already possess. Because abstract thinking does not reside solely within the domain of architecture, looking to other disciplines such as psychology and cognitive science can provide a broadening of the knowledge base in teaching abstraction in a foundation design studio. This exploration of abstraction in early, foundational design pedagogy posits that beginning students benefit from instruction in analogical reasoning, mental representation, and conceptual referents as a function of developing independent, abstract thinking skills.

Instead of causing students to perceive the disconnection between the real and the abstract, this approach relies on conceptual familiarity to bridge the mental and figurative distances between experiencing, thinking, and creating. This same phenomenon exists in other disciplines where the abstraction of information is critical to expressing intent and meaning, but the focus here is on the development of design students' selfsufficiency in creative ideation and cognitive thought instead of solely on the artifacts produced in the design process.

\section{INTRODUCTION}

Architectural education can often operate in insular patterns and pedagogical modes. In order to infuse both the academy and profession with a fresh perspective, consideration must be given to disciplines beyond architecture and, potentially, influences not typically included in and associated with the language of architectural discourse. Reyner Banham, in his essay 'A Black Box: The Secret Profession of Architecture,' described the distinctive nature of architectural education, writing, "So why do we not admit that what distinguishes architecture is not what is done...but how it is done." Here Banham emphasizes that process matters; additionally, the means and methods by which we educate architects in the process of conceptualization and abstraction also matter.
Later in the same essay, Banham notes how pedagogical insularity in architectural design can perpetuate:

Anthropologists have been known to compare the teaching studio to a tribal longhouse; the place and the rituals pursued there are almost unique in the annals of western education. One of the things that sustains this uniqueness is the frequency with which students are discouraged from pursuing modes of design that come from outside the studio.

-Reyner Banham, "A Black Box: The Secret Profession of Architecture"

Banham advocated for an interdisciplinary approach to the methodologies of making architecture, and this attitude can and should be applied to beginning design education since architects' attitudes are often formed and solidified in this early educational period. Beginning design is foundational and should be studied as its own discipline, but the individual qualities that students inherently bring to the design process should be considered as well. Perhaps the educational focus can gravitate to students' methodologies of abstract thinking instead of students viewing abstraction as merely a mysterious byproduct of the design process. Students can develop abstract thinking skills themselves, and the procedural aspects of abstraction, or the stripping away of unnecessary information, can be utilized and applied to both real and conceptual entities.

\section{IDEATION \& ABSTRACTION}

From the educator's point of view, students are often expected to be able to make the mental leap from design concept to abstraction to design representation with little familiarity with any of them. Real experience does not automatically connect to these design modes unless those connections are explicitly demonstrated to students by critics and professors. Seemingly sophisticated design output by students can mask a lack of independent thought development when the pedagogical focus is only on the resulting design object. From a cognitive science perspective, humans need to understand how they function and formulate abstract thought to enable them to repeat this thinking process later. Additionally, students must have a framework for thinking, including abstractly, for themselves in the design studio. The design goal in the foundation studio regarding abstraction should include an understanding by students of what they are doing, why they are doing it, and how they might be able to do it again. Students need not wait 

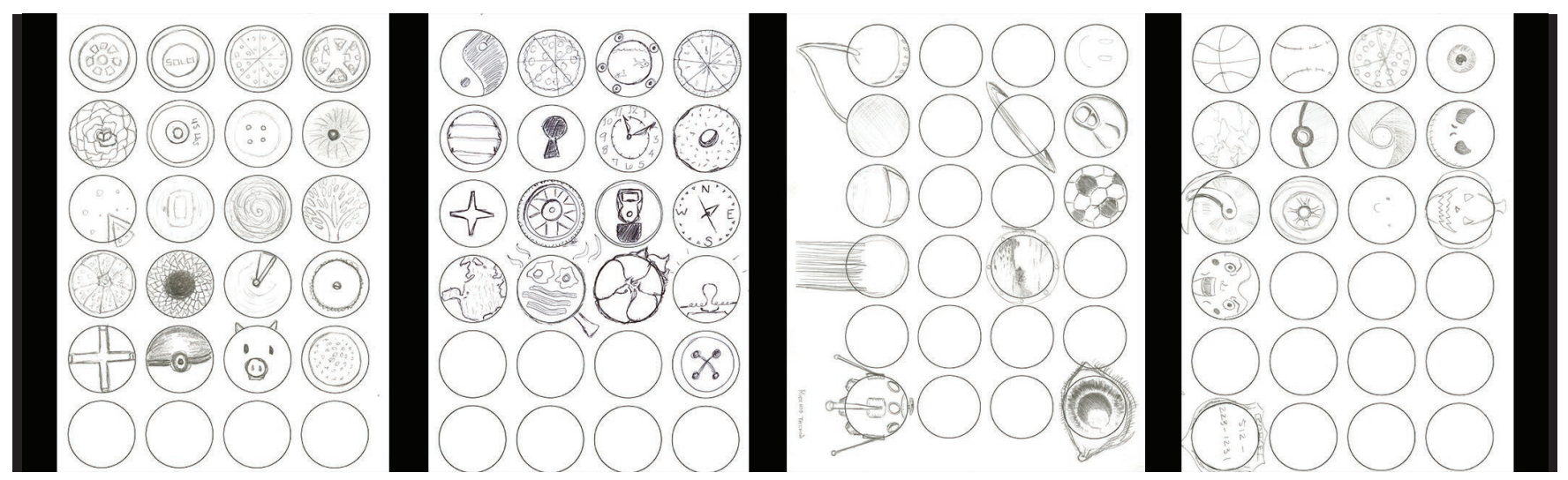

Figure 1. Fluency Class Exercise, Student Work Sample.

until later in their design education to achieve familiarity with abstraction, but instead can build upon skills already in place.

Many factors influence the human capacity for thought and ideation. In looking at issues of human cognition regarding neuroscience, it seems much is known regarding the mechanisms and functions of the brain. However, there are still seemingly endless avenues for subsequent exploration, perhaps utilizing various future technologies, to allow for the collection of data about the brain that is yet unknown. Abstraction and creativity are often linked in similar mental processes, but both are fundamental components of the design process. Creativity is defined academically as having two core characteristics: the resulting, created solution to a given problem must be both novel and useful, offering value to the problem's domain or subject matter. Another essential component of creativity is intentionality in that a truly creative solution to a given problem would be conscious, and not accidental, on the part of its creator. This demonstration of intent sounds straightforward enough, but solving a problem where elemental components or operators are missing can cause discomfort and doubt in those who engage in the creative process even when someone has previously solved a different creative problem. Because one is always searching for a novel solution, the creative process can be repeatedly daunting. Additionally, abstraction inherently has specific components removed from the design process, so making connections to reality is more complicated than assessing apparent similarity.

In teaching beginning architectural students to construct their creative processes, they experience frustration with the process even when they have previously achieved successful results with different design schemes. So how can one create a framework for solving abstract, ill-defined problems that can be useful to rely on repeatedly? Several cognitive studies point out the inherent difficulty in tackling ill-defined problems. These types of problems are overwhelming "not because we are swamped by the task of searching through an enormous number of alternative possibilities, but because we have trouble thinking of even one idea worth pursuing."
(Holyoak) This phenomenon describes the primary driver that can stymie creativity resulting in writer's block, designer's block, and other forms of problem-solving paralysis. In order to increase student capacity for ideation, low-risk class exercises designed to practice creative fluency can be implemented with beginning design students (figure 1).

\section{ANALOGICAL REASONING}

One area of cognition pertinent to architecture and the nature of abstract problem solving is the use of analogical reasoning. In a problem proposed by a now-classic cognitive study, study participants are challenged to figure out how a doctor can eradicate a tumor surrounded by healthy tissue without damaging any of this tissue. While most of the participants are unable to formulate a solution to the problem, the answer is revealed through the use of an analogy when participants are later told a story of a general who captures a fortress without destroying surrounding villages. (Duncker) This idea of making connections and applying related strategies is similar to the process of architectural design wherein the utilization of an analogy known in architectural terms as 'analogical design' assists the architect in making holistic decisions throughout the design process. The analogy can also serve as an interpretive device for visitors to the completed building in making visual, spatial and emotional connections to familiar concepts. Also, depicting analogical examples for students can demonstrate the origins of abstraction. That is, how close the analogy can mimic the actual device and still maintain a representative quality instead of an actual, literal translation of the original device is vital to understanding abstraction. In architecture, an example of this violation of the analogy and absence of abstraction would be comparing the Eiffel Tower in Paris, France and the Paris Hotel in Las Vegas (figure 2) because the second object is a no longer symbolic but instead is a direct copy of the original.

Mental representation holds a significant place in the process of human reasoning as it has the potential to be a key method of learning and processing information. In learning new concepts, people often compare new information to similar information that is already known so that one can begin 


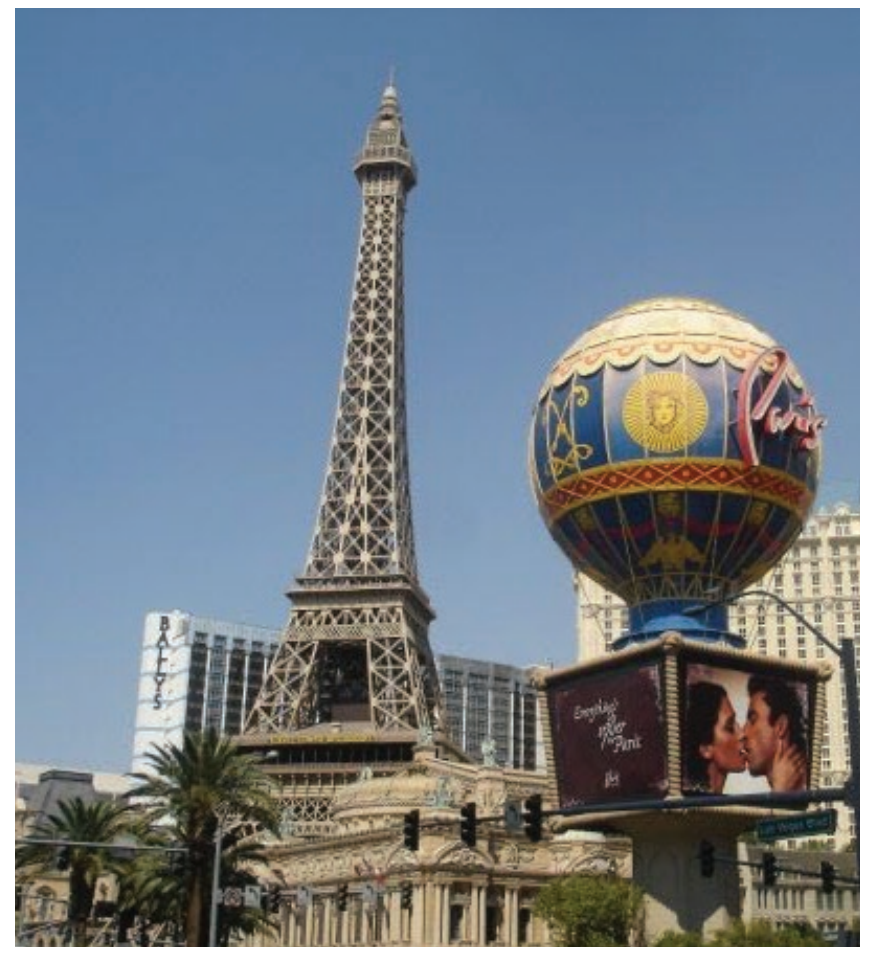

Figure 2. Paris Hotel, Las Vegas, Nevada; Copyright: TripAdvisor.

to distinguish similarities and differences in ideas. It is in this way that human learners can develop an understanding of differentiation in concept learning through analogical reasoning, a specific form of mental representation and comparison, as well as an accompanying process for acquiring new, abstract information in the future. Cognitive researchers note that "analogies are a useful form of representation because they allow us to generalize our learning." (Friedenberg, 2006) These generalized learning processes may involve diverse methods of representation such as visual, numerical and syntactic analogy in order to decipher information and prepare for operations of abstraction. Historical texts describe this phenomenon of representation in information processing through a variety of theories by thinkers and philosophers in fields as varied as architecture, design, logic, mathematics, art, and science.

Modern research in analogical reasoning has been informed by previous thinking on analogy dating as far back as antiquity and, in turn, informs research on the topic up until the present day. Beginning with Aristotle's musings on analogy, Mary B. Hesse argues in Models and Analogies in Science that "Aristotle's use of 'analogy' depends in the first place on his notion of classification of particulars into species and genera," furthering the idea that a structure for analogical reasoning is useful, and perhaps, necessary. Hesse goes on to write "that the way forward is to question, not the notion of analogy, but the notion of univocity [descriptions of God], and that both scientific arguments and ordinary language employ analogy as the normal and not the exceptional case." (Hesse, 1966) Another historical thinker on the subject of analogical reasoning, the seventeenth-century philosopher and mathematician Gottfried Leibniz, theorized that the basis for resolution of disputes between parties could be mediated through calculation. This method of problemsolving relied on a construct through which argumentation could be filtered. (Leibniz, 1898) Leibniz's writings later provided the basis for research in reasoning and problem analysis such as that of the mathematician Gottlob Frege who established a formal method for matching patterns in mathematical proofs. Frege's proofs introduced first-order predicate logic and introduced variables as a means of achieving analytical solutions via mathematical methods. (Detlefsen, 1992) A common thread that emerges historically is the need for a succinct structure for analyzing analogical thought processes. The cognitive researcher Keith Holyoak published research that also introduced a "theoretical framework for understanding analogical thinking and its relationship to the acquisition of schemas." (Sternberg, 1984) The use of language descriptors in analogical problems is useful for beginning design students in architecture since language skills are already in place, and students do not need to wait for drawing and digital skills to develop before understanding relational concepts.

\section{STRUCTURE-MAPPING \& REPRESENTATION}

The contemporary work of cognitive scientists, through their work in cognitive science and analogical reasoning, builds on the foundation laid by earlier thinkers in cognition. The 1983 paper titled "Structure-Mapping: A Theoretical Framework for Analogy" by Dedre Gentner provided a set of rules for analogical comparison and established a unified process of analysis to clarify the relationship of research areas as disparate and varied as architectural theory and abstract design. "Structure-Mapping" served as a benchmark in the field of analogy, and it is considered a classic paper because it laid out the prescriptive outline of structure-mapping theory, "the interpretation rules (of which) are characterized as implicit rules for mapping knowledge about a base domain into a target domain." (Gentner, 1983) Any theory of analogy might describe how hidden meaning is derived from the meaning of a known quantity. Analogies are often referenced in architectural education, but here structure-mapping outlines a specific theory that establishes interpretation rules for analogical reasoning by clarifying the overall meaning of the analogy. Analogy in this sense does not rely on the number of shared vs. non-shared features; that is, the "degree of overlap" is not valued. These rules are clarified as follows:

Two important features of the theory are (a) the rules depend only on syntactic properties of the knowledge representation, and not on the specific content of the domains; and (b) the theoretical framework allows analogies to be distinguished cleanly from literal similarity statements, applications of abstractions, and other kinds of comparisons.

-Dedre Gentner, "Structure-Mapping: A Theoretical Framework for Analogy" 

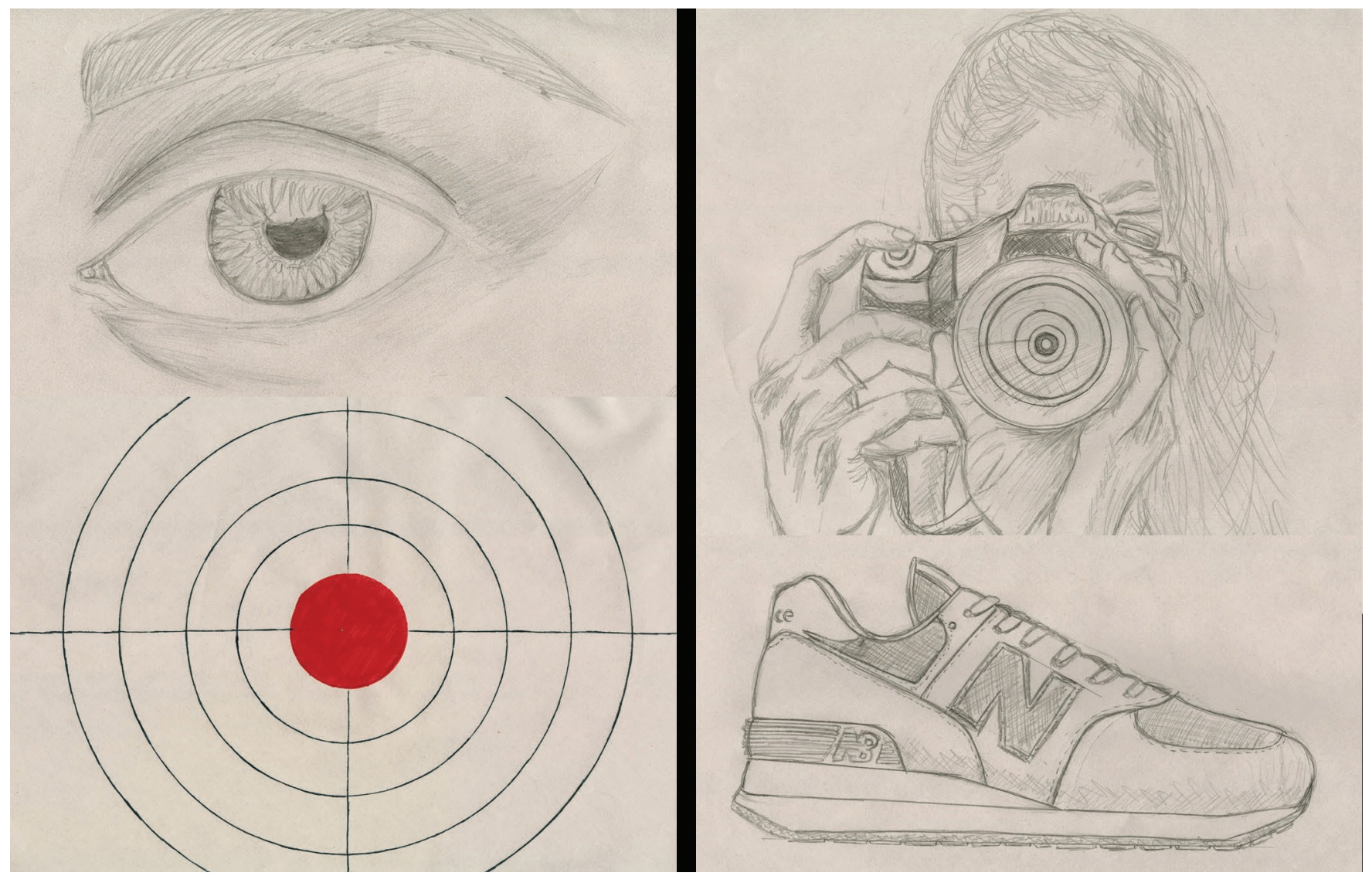

Figure 3. Structure-Mapping Assignment, Student Work Sample.

Theoretical rules dictate that relationships between objects are paramount, and the individual object attributes or qualities are irrelevant. So, the simple steps of the theory dictate one must first discard attributes of objects, then try to preserve relations between objects, and third, decide which relations are preserved and choose the systems of relations: the systematicity principle. The comparison of a battery to a reservoir illustrates this process:

The essence of the analogy between batteries and reservoirs is that both store potential energy, release that energy to provide power for systems, etc. We can be quite satisfied with the analogy in spite of the fact that the average battery differs from the average reservoir in size, shape, color, and substance.

-Dedre Gentner, "Structure-Mapping: A Theoretical Framework for Analogy"

This example demonstrates the application of simple rules across disciplines where the distinction of comparison types is valued. The rules of systematicity are also distinct in that they prescribe the differences between literal similarity, analogy, abstraction, and anomaly. The long-standing legacy of the "Structure-Mapping" paper is the transformation of the structure-mapping theory into the structure-mapping engine including the demonstration of the theory utilizing simple diagrams to depict object-relations. Many other cognitive researchers have more recently created digital platforms in the vein of the structure-mapping engine to analyze comparable properties across a variety of disciplines. An architectural application of this principle can be seen in figure 3, a beginning design assignment wherein students must make visual comparisons to a given object in the form of: analogy, anomaly, and literal similarity.

Mental representations are critical for learning new information, and the principles of mapping in analogical reasoning offer a stable framework for assessing comparison types. Relational properties define an analogy, and these relationships are applicable across disciplines including in architecture. Analogical architectural design principles can translate to other creative fields, but it will take further research by those within the architectural academy to cross into those disciplines as cognition research can offer a substantive vehicle for defining criteria in other disciplines. An analogy in architectural design or architectural theory is often employed to establish a relationship with concepts or schema. Analogical design can, and often does, reference an abstraction of the design idea. Analogy may be manifest in contextual referents with case study examples in architectural education. Architectural education would benefit from 


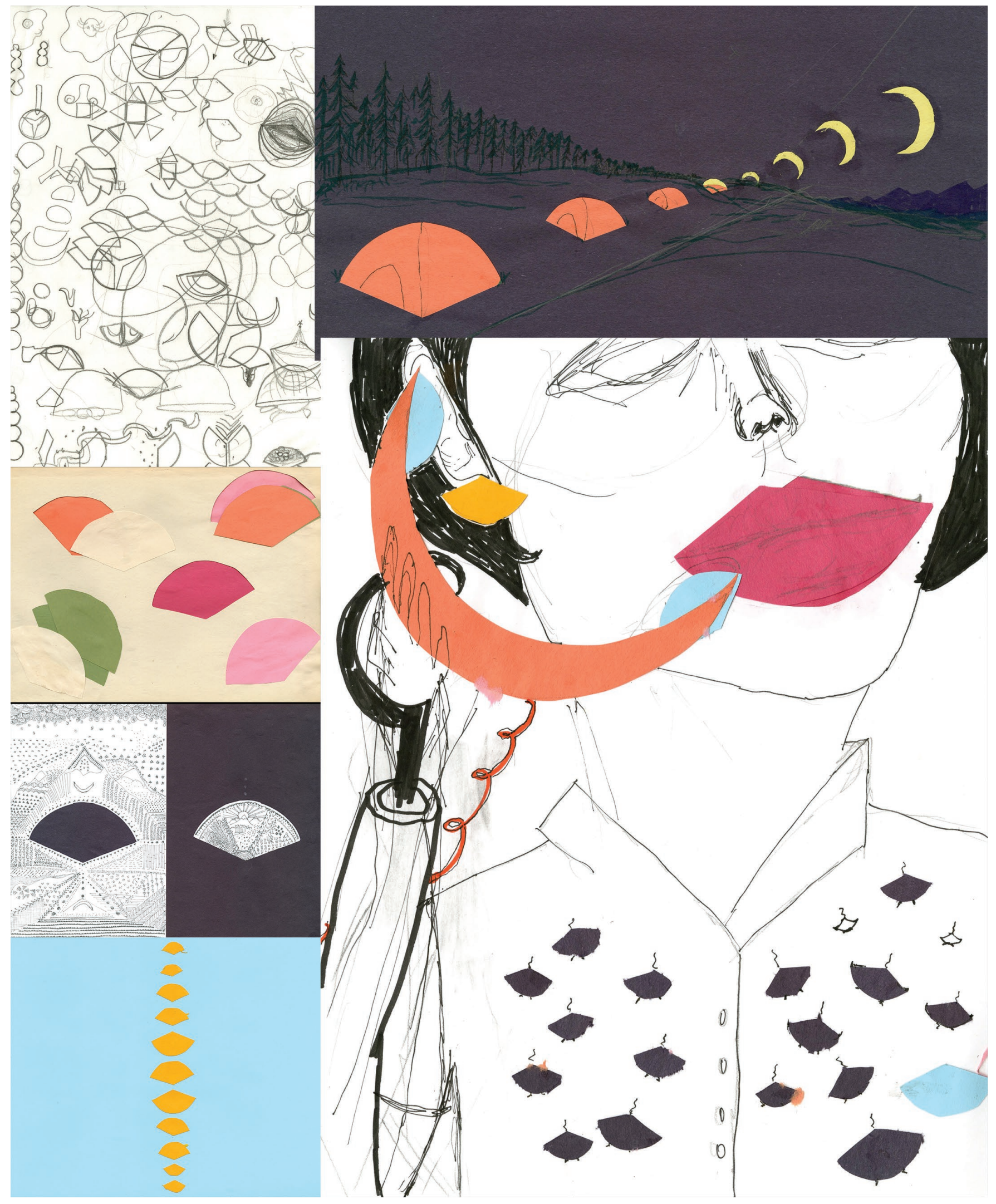

Figure 4. Pattern-Making Assignment, Student Work Sample. 
a greater influence from other disciplines generally, and from areas of cognitive science. For example, architectural analysis could benefit from a more structured approach to defining an analogical reference such as the set of simple rules set by the concept of structure-mapping. One potential practical application for an analogical framework in architecture include the ability to define boundaries for students of architecture as they establish criteria for analogical referents in their designs.

\section{VISUAL PROCESSING \& PERCEPTION}

As the practice of architecture currently exists, analogical design in architecture often lacks a theoretical structure for quantifiable outcomes so the definitions for analogy, similarity, and abstraction are blurred and confused within the practice of architecture and architectural education. This likely remains the case within architecture because the profession can be insular with little cross-disciplinary academic research taking place. The practice and theories within architecture can be advanced in technological ways, but sometimes not in philosophical ones. Other aspects of cognitive science besides analogy that could benefit architecture include perception and vision theory in addition to many others. Many principles of cognitive science haven't made their way into the practice of architecture, even in some aspects of design ideation though some similarities may already exist in diagramming depictions. Architectural theorists look to other disciplines including psychology but would likely benefit from further explorations into cognitive science.

In contemporary research about the cognitive area of interactive vision, cognitive scientists argue for a rethinking of traditional approaches to the study and understanding of visual processing. In its most orthodox form, prior assumptions regarding vision have isolated its study and task-related research from other sensory processes with this prevailing school of thought labeled as the 'Theory of Pure Vision.' Current researchers lay out the case for adopting what they refer to as 'interactive vision' to address and explore the influence of other sensory functions on vision in a more holistic research framework. One interesting connection can be made between this interactive strategy and learning as the way in which humans take in information. Animals and humans learn about the world around them through their sensory perception including their visual computation and can evolve based on what they discover. So it stands to reason that "evolution found the interactive strategy good for brains" (Friedenberg, 2006). The brain learns patterns that go together, through information gathered via visual intake, then adapts to reduce the time needed to re-process this information later. This mode of pattern-making could also be effective for creative, abstract processes such as architectural design if visual connections were made into patterns early on that could be skipped over in order to adapt to learning higher-level processing. The same phenomenon exists in artistic disciplines where visual intake of information is critical to learning skills, but also the other way around. That is, a skill such as drawing is seen as a metacognitive one because someone is learning to draw while also learning visually about the item they are drawing. Then, when one draws a similar item, later on, one does not need to visually focus or physically "see" the same level of detail because the brain has become accustomed to its representation and how it should look.

A related topic of cognitive research whose understanding could benefit the discipline of architecture is perception. The study of object perception by modern perception researcher Elizabeth Spelke sought to clarify standardized principals of understanding in the areas of perception and perceptual development in human infants and to identify the specific means by which humans perceive objects. A research study Spelke conducted offered two proposals: one hypothesized that when perception grows as a result of experience, then perceptual abilities are enhanced but not altered in a fundamental way. The other proposal proffered a methodological study that identified the origins of perception, thus informing aspects of adult perception. The unifying element of the study centered on "the ability to organize arrays of surfaces into unitary, bounded, and persisting objects." (Spelke, 1990) To understand the impetus for this research, the context of this study involved the existence of two competing schools of thought on the subject. The first involved Empiricist theories which held that perception is limited to what can be taken in by the senses and cannot be known before experience. The other competing view was that of the Gestalt theorists, a group of German psychologists whose theorems focused on mental operations instead of sensory ones. The term 'gestalt' refers to the wholes these theorists viewed as more than the sum of its parts with the compounded object called the 'Pragnanz' or 'good figure.' (Friedenberg, 2006)

Through an analysis of Gestalt psychology and disciplines within cognitive science, research in abstraction touches on related aspects of phenomenology and perception which bridges to other disciplines outside of cognitive science. It is this philosophical approach that is most prevalent in architecture. In architecture, phenomenology as experiential perception is studied and academically examined in the context of the built environment. Here, the perceived objects are buildings as the uninitiated observer and their perceptions encounter them. However, phenomenological psychology can be applied to architectural education via 


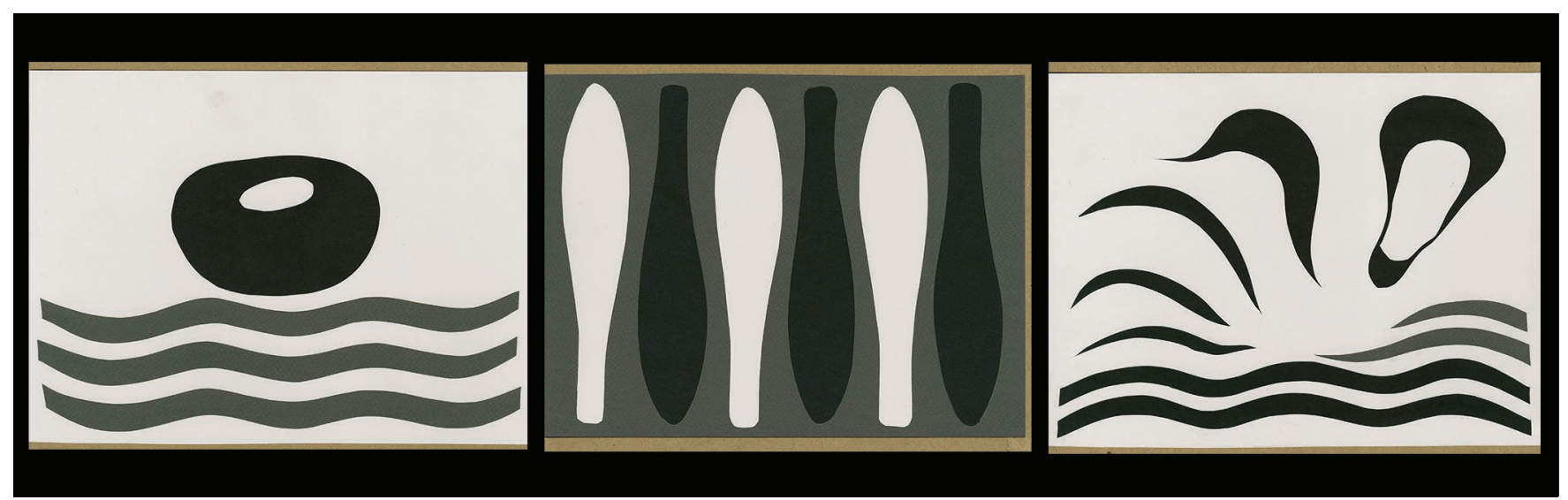

Figure 5. Figure/Ground Assignment, Student Work Sample.

perception through the act of building design and creation. It is in this way that the bodily experience of making the models and drawings for the building's design can inform future perceptions of the built work. Additionally, the designer may attempt to convey an intentional manipulation of object perception and depth perception through the juxtaposition of object clusters such as figure/field, figure/figure, and figure/ ground (see figure 5), not unlike the figures used in other types of cognitive studies.

\section{CONCLUSION}

Are current methodologies in early architectural education helping or hurting to address and develop abstract thinking? In an effort to introduce rigor through layers of regimented instruction and project steps, it is possible to make the project process far too prescriptive to engage in abstraction. The first semesters and year of design education likely form a significant imprint on the remainder of a student's educational experience. Explaining and developing skills in abstraction and conceptualization is paramount in assisting students to make choices and eliminate extraneous design elements. Interdisciplinarity and familiarity are critical to understanding abstraction in beginning design even though architecture merely is one of many disciplines that utilizes abstract thinking. Reyner Banham echoes the need for looking to other disciplines for guidance concerning architectural pedagogy:

It (architecture) could permit itself to be opened up to the understandings of the profane and the vulgar, at the risk of destroying itself as an art in the process. Or it could close ranks and continue as a conspiracy of secrecy, immune from scrutiny, but perpetually open to the suspicion, among the general public, that there may be nothing at all inside the Black Box except a mystery for its own sake.

-Reyner Banham, "A Black Box: The Secret Profession of Architecture"
Jane Rendell reinforces this approach to architecture and disciplinarity in 'Architectural research and disciplinarity' noting:

Many argue against architecture's uniqueness since they believe this marginalises architecture within the academy and strengthens the perception that architecture holds a fragile compatibility with other models of academic research. If we choose instead to argue that architecture is unique, on what grounds can we make such a statement?

-Jane Rendell, "A Black Box: The Secret Profession of Architecture"

The concept of the 'Black Box' offers endless possibilities for modes of instruction in architectural pedagogy, and a wide range of approaches exists for promoting higher levels of understanding in beginning design students.

\section{REFERENCES}

Detlefsen, Michael, ed. Proof and Knowledge in Mathematics. New York: Routledge, 1992.

Friedenberg, Jay \& Gordon Silverman. Cognitive Science: An Introduction to the Study of Mind. Thousand Oaks, CA: Sage Publications, 2006.

Gentner, Dedre, Keith J. Holyoak, and Boicho N. Kokinov, eds. The Analogical Mind: Perspectives from Cognitive Science. Cambridge, MA: The MIT Press, 2001.

Gentner, Dedre. "Structure-Mapping: A Theoretical Framework for Analogy." Cognitive Science 7, no. 2 (1983): 155-170.

Hesse, Mary B. Models and Analogies in Science. Notre Dame, IN: University of Notre Dame Press, 1966.

Leibniz, Gottfried Wilhelm. The Monadology and Other Philosophical Writings, trans., Robert Latta. Oxford: Clarendon Press, 1898.

Sternberg, Robert J., ed. Advances in the Psychology of Human Intelligence Volume 2. Hillsdale, NJ: Lawrence Erlbaum Associates, 1984. 